\title{
Computational homogenization of unsteady incompressible flows with obstacles
}

\author{
M. Shakoor* and C.H. Park \\ IMT Lille Douai, Institut Mines-Télécom, Univ. Lille, Centre for Materials and Processes, \\ F-59000 Lille, France \\ Email: modesar.shakoor@imt-lille-douai.fr
}

\begin{abstract}
Computational homogenization and its Finite Element (FE) implementation is now well understood and widely used to model the multiscale mechanics of materials. An FExFE or FE simulation relies on an FE model of a coarse domain which embeds other FE models of a fine domain at each of its integration points [1]. The fine domain should be a Representative Volume Element (RVE) capturing the fine aspects of the heterogeneity to be homogenized, so that the coarse domain can be modeled as homogeneous. This approach is relevant if the characteristic length of the heterogeneity is very small compared to that of the coarse domain, as it avoids representing this heterogeneity directly within the coarse FE mesh.
\end{abstract}

The scale transition requires a mathematically sound homogenization theory providing the equations to solve at the coarse scale, and the boundary conditions to use at the fine scale [2]. There is a significant amount of litterature on $\mathrm{FE}^{2}$ methods in solid mechanics, but not in fluid mechanics. One exception is steady flows through porous media, which are relevant for reservoir engineering and composites moulding applications among others [3].

In a recent study, it has been shown that computational homogenization is possible for steady incompressible flows with obstacles at the fine scale, even with the autoadvection term of the Navier-Stokes equations [4]. In this presentation, it is proposed to go even further and address unsteady flows.

To reach this objective, several challenging problems will be solutioned:

- First, it will be necessary to handle the incompressibility constraint both at the coarse and fine scales. Compatible FE discretization will be proposed to avoid instability.

- Then, it will be necessary to implement a two-way coupling between the coarse and fine scale models. At each time increment, a nonlinear problem will be solved using an iterative Newton-Raphson procedure at the two scales. Coarse scale variables interpolated at integration points will provide boundary conditions for the fine scale problems. Fine scale solutions averaged over the RVEs will provide the terms of the equations to solve at the coarse scale.

- One challenging aspect of the two-way coupling will be the computation of the tangent modulii for the linearization of the coarse scale equations. Several automatic differentiation procedures will be introduced for this operation.

This original $\mathrm{FE}^{2}$ approach for unsteady flows will be implemented in the FEMS open-source software [5], [6]. Numerical results will be presented to demonstrate its accuracy with and without obstacles within the RVE.

\section{ACKNOWLEDGMENTS}

The authors would like to acknowledge for the financial support from the French National Research Agency (ANR) to the project "COMP3DRE" (grant number: ANR-16-CE08-0042). 


\section{REFERENCES}

[1] F. Feyel, "Multiscale FE2 elastoviscoplastic analysis of composite structures," Comput. Mater. Sci., vol. 16, no. 1-4, pp. 344-354, Dec. 1999, doi: 10.1016/S09270256(99)00077-4.

[2] P. J. Blanco, P. J. Sánchez, E. A. de Souza Neto, and R. A. Feijóo, "Variational Foundations and Generalized Unified Theory of RVE-Based Multiscale Models," Arch. Comput. Methods Eng., vol. 23, no. 2, pp. 191-253, Jun. 2016, doi: 10.1007/s11831-014-9137-5.

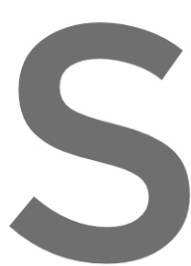

[3] C. Sandstöm, F. Larsson, and K. Runesson, "Weakly periodic boundary the homogenization of flow in porous media," $A d v$. no. 1, p. 12, I

[4] P. J. Blanco, equations by Appl. Mech.

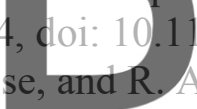
86/s40B23-014-001 A. Feijor
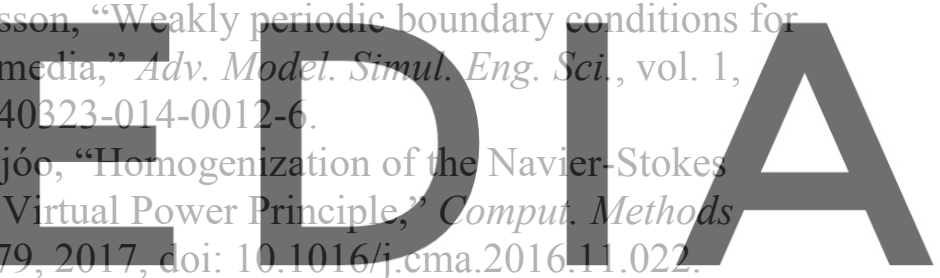

[5] M. Shakoor, "FEMS - A Mechanics-oriented Finite Element Modeling Software,"

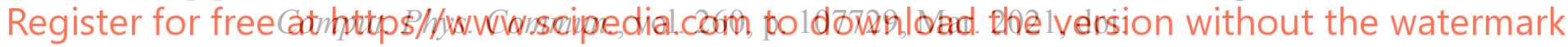
10.1016/j.cpc.2020.107729.

[6] M. Shakoor and C. H. Park, "A higher-order finite element method with unstructured anisotropic mesh adaption for two phase flows with surface tension," pp. 1-29, Oct. 2020, [Online]. Available: http://arxiv.org/abs/2010.13716. 